\title{
3 Mehrdeutigkeiten sprachlicher Ausdrücke
}

Untersuchungen zur Mehrdeutigkeit sprachlicher Ausdrücke haben eine lange Tradition. Schon die Stoiker wussten um diese Bedeutungsvielfalt, darum, dass ein einzelner Gedanke auf unterschiedlichen Wegen ausgedrückt werden kann und einzelne Wörter verschiedene Bedeutungen haben (vgl. Ravin/Leacock 2000: 1). Bereitet das Vorliegen unterschiedlicher Verwendungsweisen eines Ausdrucks Sprechern in der alltäglichen Praxis normalerweise keine großen Probleme, ist die lexikalische Ambiguität sprachlicher Ausdrücke aus Sicht von Linguisten mit einer Vielzahl theoretischer und methodischer Fragen verbunden (ebd.), die auch in anderen Fachrichtungen - z. B. der Lexikographie, der Computerlinguistik, der Übersetzungswissenschaft, der Psycholinguistik, der Künstliche-Intelligenz-Forschung, der Spracherwerbsforschung sowie der Sprachdidaktik - diskutiert werden ${ }^{72}$. Für einen kurzen Überblick mit Literaturangaben siehe Clark/Nerlich (2003).

Sprachliche Ausdrücke, die mehr als eine wörtliche Bedeutung haben, werden im Allgemeinen mit dem Terminus Polysemie ${ }^{73}$ (griech. ,Vieldeutigkeit') charakterisiert (vgl. z. B. Taylor 2003b: 32; Evans 2005: 33). Polysemie gilt i.d.R. „as the normal order of things in natural language“ (Taylor 1993: 151f.). Schlägt man beispielsweise das Verb stellen im Duden - Das große Wörterbuch der deutschen Sprache (2012 $)$ nach, ergibt sich folgendes Verwendungsweisenspektrum:

stellen

1 a sich an einen bestimmten Platz, eine bestimmte Stelle begeben und dort für eine gewisse Zeit stehen bleiben

b an einen bestimmten Platz, eine bestimmte Stelle bringen und dort für eine gewisse Zeit in stehender Haltung lassen; an einer bestimmten Stelle in stehende Haltung bringen

\footnotetext{
72 Die aktuelle Polysemieforschung beschäftigt sich mit Fragen wie Welcher Art sind die unterschiedlichen Bedeutungen der einzelnen Lexeme?, Wie grob- oder feinkörnig müssen die Bedeutungen unterschieden werden?, Wie hängen die unterschiedlichen Bedeutungen zusammen?, Wie lassen sich die unterschiedlichen Verknüpfungen zwischen den Verwendungsweisen ermitteln? (vgl. Taylor 2003a; Bones 2009). Bei der Sichtung aktueller Arbeiten der Polysemieforschung zeigt sich, dass „the last word on polysemy has not yet been spoken“ (Taylor 2003a: 654).

73 Der moderne Begriff der Polysemie geht auf den französischen Semantiker Michel Bréal (1832-1915) zurück. Es ist jedoch zu berücksichtigen, dass - wie oben bereits angedeutet - ,the ,multiplicity of meaning', with or without the label „polysemy“, was quite well researched [...] well before Bréal“ (Nerlich 2003: 49). Eine gute Darstellung zur Entwicklung des Polysemiebegriffs findet sich u.a. in Nerlich (2003). Umfassende Darstellungen zum gegenwärtigen Stand der Polysemieforschung finden sich in den Sammelbänden Ravin/Leancock (2000); Cuyckens/ Zawada (2001) und Nerlich/Clarke (2003).
}

๖ Open Access. ( 2021 Tanja von der Becke, publiziert von De Gruyter. (c) BY Dieses Werk ist lizensiert unter einer Creative Commons Namensnennung 4.0 International Lizenz.

https://doi.org/10.1515/9783110726268-003 
2 etwas an einen bestimmten Platz, eine bestimmte Stelle bringen, tun [sodass es dort steht]

3 (von Fanggeräten) aufstellen

4 (von technischen Einrichtungen, Geräten) in die richtige oder gewünschte Stellung, auf den richtigen oder gewünschten Wert o. Ä. bringen, so regulieren, dass sie zweck-, wunschgemäß funktionieren

5 dafür sorgen, dass jemand, etwas zur Stelle ist; bereitstellen

6 einen bestimmten Zustand vortäuschen

7 (von Speisen, Getränken) etwas an einen dafür geeigneten Platz stellen, damit es eine bestimmte Temperatur behält oder bekommt

8 zum Stehenbleiben zwingen und dadurch in seine Gewalt bekommen

9 a (von jemandem, der gesucht wird, der eine Straftat begangen hat) sich freiwillig zur Polizei o. Ä. begeben, sich dort melden

b um einer Pflicht nachzukommen, sich bei einer militärischen Dienststelle einfinden, melden

c einer Herausforderung o. Ä. nicht ausweichen; bereit sein, etwas auszutragen

10 sich in bestimmter Weise jemandem, einer Sache gegenüber verhalten; in Bezug auf jemanden, etwas eine bestimmte Position beziehen, Einstellung haben

11 jemandem ein bestimmtes Auskommen verschaffen

12 (Kaufmannssprache, besonders österreichisch) einen bestimmten Preis haben, eine bestimmte Summe kosten

13 (in Bezug auf die Stellungen und Bewegungen der Personen [auf der Bühne]) festlegen; arrangieren

14 steif in die Höhe richten, aufstellen

15 aufgrund bestimmter Merkmale, Daten o. Ä. erstellen, aufstellen

16 in verblasster Bedeutung in Verbindung mit bestimmten Substantiven

Kennzeichnend ist, dass zwischen den einzelnen Bedeutungsvarianten des Verbs stellen bestimmte, nicht-triviale Beziehungen bestehen (vgl. Dölling 2005). Entsprechend wird Polysemie normalerweise definiert als ,the association of two or more related senses with a single linguistic form“ (Taylor 1995a: 99). Hiervon zu unterscheiden ist die sog. Homonymie (griech. ,Gleichnamigkeit‘), bei der vereinfacht ausgedrückt - zwei oder mehr Lexeme mit verschiedenen Bedeutungen zufällig die gleiche Form haben. Polysemie und Homonymie werden also i.d.R. als zwei Arten der lexikalischen Ambiguität aufgefasst, die sich vor allem 
hinsichtlich ihrer systematischen Verknüpfung bzw. zufälligen Formgleichheit unterscheiden. Die Grenze zwischen Homonymie und Polysemie ist jedoch nicht immer eindeutig, da bei den verschiedenen Abgrenzungsversuchen auf unterschiedliche Kriterien zurückgegriffen wird - etwa auf die vier Dimensionen

(a) etymological level (historical relatedness), (b) semantic dimension (synchronic relatedness of meaning), (c) grammatical dimension (belonging to the same or to different grammatical classes), and (d) representational dimension (representation by distinct subentries or by distinct main entries).

(Behrens 2002: 321)

Weitestgehende Einigkeit in der Polysemieforschung besteht aber darin, dass dem etymologischen und dem semantischen Kriterium besondere Bedeutung zur Unterscheidung von homonymen und polysemen Ausdrücken zukommt. Keller/ Kirschbaum (2003) schreiben:

Als Unterscheidungskriterium wird im Allgemeinen das Wissen um die etymologische Verwandtschaft angesehen: Wenn ein Wort zwei systematisch unterschiedene Bedeutungen hat und die eine historisch von der anderen ableitbar ist, und wenn diese Bedeutungsverwandtschaft im allgemeinen Sprachbewusstsein präsent ist, so spricht man von Polysemie. Wenn zwei verschiedene Wörter, die keine (erkennbare) gemeinsame sprachgeschichtliche Herkunft haben, gleich lauten, so liegt Homonymie vor.

(ebd. 103)

Als das entscheidende Kennzeichen der Polysemie haben wir oben herausgestellt, dass die Bedeutungsvarianten eines Lexems miteinander verwandt sein müssen. Nicht metaphorisch ausgedrückt: Zwischen den Bedeutungsvarianten muss eine semantische Relation (z. B. metaphorischer, metonymischer und differenzierender Art) bestehen, die intersubjektiv nachvollzogen werden kann. Anders verhält es sich bei der Homonymie: Hier handelt es sich um zwei verschiedene, zufällig gleich lautende Lexeme, deren Bedeutungen eben keine derartige semantische Beziehung aufweisen.

(ebd. 108)

Die Anwendung dieser und ähnlicher Kriterien ist jedoch nicht unproblematisch. So führen u. a. die Tatsache, dass für die Untersuchung historischer Sprachzustände nicht immer genügend Belegmaterial vorliegt, um die Zusammenhänge aus diachronischer Sicht zu rekonstruieren, sowie mangelndes Wissen um sprachgeschichtliche Zusammenhänge im allgemeinen Sprachbewusstsein dazu, dass eine scharfe Grenzziehung zwischen homonymen und polysemen Ausdrücken nicht immer erreicht werden kann (vgl. Bons 2009). Darüber hinaus kann eine Überprüfung anhand der zwei Kriterien auch zu unterschiedlichen Ergebnissen führen. Es kommt vor, dass sprachliche Ausdrücke aufgrund nicht mehr nachvollziehbarer Zusammenhänge zwischen den Verwendungsweisen synchron betrachtet als voneinander unabhängige Homonyme gewertet und entsprechend in Wörterbüchern als unterschiedliche Einträge gelistet werden. Ein auf den ersten Blick eindeutiges Beispiel hierfür ist das Wort Bank, das einerseits als Bezeichnung für eine Sitzgelegenheit $\left(B a n k_{1}\right)$, andererseits als Bezeichnung für ein Kreditinstitut (Bank $)_{2}$ verwendet wird. Die Annahme, 
dass es sich bei beiden Wörtern um zwei verschiedene und voneinander unabhängige Wörter handelt, scheint durch das unterschiedliche morpho-syntaktische Verhalten von Bank $k_{1}$ und Bank 2 - hier das Vorliegen unterschiedlicher Pluralformen Bänke vs. Banken - bestätigt (vgl. u. a. DUDEN 20124). Diachron betrachtet zeigt sich jedoch, dass durchaus eine etymologische Verwandtschaft zwischen den beiden Wörtern besteht (vgl. Kluge 2002 24 : 88). So ist

${ }^{2}$ Bank f. ,Geschäft für Geldverkehr', in seiner Herkunft identisch mit ${ }^{1}$ Bank (s. d.) (Anm. verf. Sitzmöbel für mehrere), das früh in die roman. Sprachen eindringt. Ital. banco m. (Nebenform banca f.), anfangs (wie mhd. wehselbanc) der Tisch der Geldwechsler, wird im 15. Jh. rückentlehnt, wobei die ital. Lautung Bancho, Ban(c)ko lange beibehalten wird. Nach frz. banque f. begegnen im 18. Jh. Pluralformen wie Banques, Banquen, unter deren Einfluß bei Anlehnung an ${ }^{1}$ Bank sich die heutige Form und das feminine Genus durchsetzen. Auf frz. Vorbild beruht auch die Verwendung von Bank als ,Spielbank im Glücksspiel. - Banknote f. Die Prägung engl. banknote (17. Jh.) wird im 18. Jh. ins Dt. übernommen, ältere Bezeichnungen wie Bankbillet, Bankzettel ablösend. ${ }^{74}$

(DWDS_EtyW_Bank; 28.05.2018)

Aufgrund solcher Abgrenzungsschwierigkeiten wurde schon häufiger vorgeschlagen, auf die Unterscheidung zwischen Homonymie und Polysemie zu verzichten (vgl. z.B. Strauß/Zifonun 1985: 220f.; Wichter 1988: 15ff., 91). Mit Verweis auf unstrittige Fälle, sprachübergreifende Vergleiche sowie darauf, „dass die Unterscheidung in hohem Maße der Intuition entspreche, wird jedoch im Allgemeinen dafür plädiert, an der Unterscheidung an sich festzuhalten“ (Bons 2009: 11). Die strikte Dichotomie zwischen Homonymen und Polysemen wird dabei oft zugunsten eines Kontinuums, auf dem Verwendungsweisen mit unterschiedlichen Graden an Distinktheit und Familienähnlichkeit angesiedelt sind, aufgehoben (vgl. Nerlich/Clarke 2003: 10).

Weil hinreichende Kriterien zur Unterscheidung von Homonymie und Polysemi $^{75}$ nicht gefunden werden konnten, verlagerte sich die Diskussion im Rahmen der Polysemieforschung zunehmend auf die methodische Fragestellung, ob sich die vielfältigen Verwendungsmöglichkeiten eines Ausdrucks auf eine (abstrakte)

74 ,DWDS_EtyW_Bank; 28.05.2018“ steht hier für die vollständige Angabe „Bank“, In: Pfeifer et al. (1993): Etymologisches Wörterbuch des Deutschen; digitalisierte und von Wolfgang Pfeifer überarbeitete Version im Digitalen Wörterbuch der deutschen Sprache, <https://www.dwds.de/ wb/Bank>, abgerufen am 28.05.2018. Im Folgenden wird nur noch die entsprechende Kurzform (DWDS_EtyW_ [Verb; Abrufdatum]) verwendet.

75 Darüber hinaus merkt Taylor (2003a: 645) zu Recht an, dass eine „speaker's metalinguistic awareness of whether two senses of a word are related or not most likely has no consequences at all for the way the person uses the word. It is sufficient simply that the speaker has learned the appropriate facts of usage.“ 
Grundbedeutung zurückführen lassen oder ob man besser mehrere eigenständige ggf. miteinander verknüpfte Verwendungsweisen annehmen sollte.

\subsection{Bedeutungsvarianten als Ausdruck von Polysemie}

In der methodischen Debatte werden zentrale Fragen, u. a. bezüglich der Einheit der Bedeutung sowie der Frage nach dem Gegenstand der Semantik in Abgrenzung zur Pragmatik, aus verschiedenen bedeutungstheoretischen Perspektiven betrachtet. So hat aus Sicht eines Strukturalisten, der das

one form, one meaning“-Prinzip [...] vertritt, [...] die Diskussion unter Umständen andere Schwerpunkte als aus Sicht eines Kognitivisten, dem es um die Frage nach der mentalen Repräsentation von Bedeutung geht, oder aus der Sicht eines Handlungstheoretikers, für den der tatsächliche Gebrauch eines Ausdrucks Ausgangspunkt ist.

(Bons 2009: 16)

Diein dieser Diskussion vertretenen Positionen werden in der Literatur als (i) monosemic approach (auch: single-meaning-approach), (ii) homonymic approach und (iii) polysemic approach bezeichnet (vgl. Lewandowska-Tomaszczyk 2007). Vertreter des monosemic approach (z. B. Bierwisch 1983; Lang 1993; Pustejovsky 1995; Pohl 2010) gehen davon aus, „dass die Bedeutungsvarianten eines mehrdeutigen Ausdrucks auf eine einheitliche unterspezifizierte Kernbedeutung zurückgeführt werden“ können ${ }^{76}$ (Pohl 2010: 173). „Erst wenn diese unterspezifizierte Grundbedeutung mithilfe konzeptuellen Wissens weiter spezifiziert bzw. semantisch angereichert ist, gelangt man über mehrere Schritte zu einer Kontext- oder Äußerungsbedeutung.“ (ebd.) Im Rahmen der Monosemie- oder Kernbedeutungshypothese ${ }^{77}$ lassen sich also die unterschiedlichen Lesarten eines „Verbs -

76 Der Versuch, Polysemie tendenziell auf Monosemie zu reduzieren, gewinnt seit der Einführung der formalen Pragmatik - und hier vor allem seit der Entwicklung der Implikaturtheorie von Grice (1969) - zunehmend an Bedeutung.

77 Bei der Frage, ob es sich bei den verschiedenen Verwendungsmöglichkeiten eines sprachlichen Ausdrucks um Variationen eines monosemen Ausdrucks (Monosemie) oder um mehrere eigenständige, u. U. miteinander verwandte Verwendungsweisen (Polysemie) handelt, wird häufig auch auf den Begriff der Vagheit zurückgegriffen. Während mit dem Begriff der Monosemie hervorgehoben wird, dass für einen sprachlichen Ausdruck mehrere Verwendungsweisen angenommen werden, wird mit dem Begriff der Vagheit (auch: lack of specification bzw. Unterspezifikation verwendet (vgl. u.a. Kempson 1977; Dölling 2005)) stärker betont, dass beim Gebrauch dieser Verwendungsweisen einzelne Aspekte offen bleiben (vgl. Bons 2009: 13).

Daneben wird der Begriff der Vagheit auch im Zusammenhang mit Konzepten verwendet, „die Merkmale beinhalten, deren Wert auf einer kontinuierlichen Skala oder in einem kontinuierlichen Bereich variieren kann“ (Löbner 2015²: 56). Danach sind z. B. Farbwörter wie grün 
ausgehend von einer einheitlichen, schematischen Basisbedeutung - als Resultat seiner konzeptuellen Differenzierung im Kontext“ (Dölling 2005: 177) erfassen, wobei die Festlegung auf eine Bedeutungsvariante erst durch die Einbettung des semantisch unterspezifizierten Verbs in einen sprachlichen und pragmatischen Kontext erfolgt. Polysemie ist demnach ,a consequence of alternative conceptual interpretations of a unitary semantic representation“ (Taylor 1995b: 3). Durch die Trennung semantischer und konzeptueller Prinzipien soll die Gefahr einer „Polysemie-Inflation“ (Herweg 1988: 55) vermieden und somit eine größtmögliche Ökonomie des mentalen Lexikons angestrebt werden.

Die extreme Gegenposition hierzu bildet der sog. homonymic approach. Vertreter der Homonymie-Hypothese (vgl. Kempson 1977) versuchen, „alle nur denkbaren Varianten, sei es nun, daß sie sich in semantischer oder syntaktischer Hinsicht unterscheiden, als einzelne Bedeutungen aufzuführen und somit polyseme Lexeme im Prinzip wie Homonyme zu behandeln“ (Chur 1996: 15). Die einzelnen Verwendungsweisen der Lexeme bilden nach dieser Auffassung „simply a bizzare accident with each meaning being completely unrelated to each other“ (Evans 2004: 87). Entsprechend müssen die Bedeutungen einzeln und voneinander unabhängig gelernt und von den jeweiligen Sprechern und Hörern in der konkreten Verwendungssituation aus den gelernten Varianten ausgewählt werden.

Keine der beiden Extrempositionen, weder der monosemic approach noch der homonymic approach, kann aus empirischer Sicht jedoch überzeugen ${ }^{78}$. Es ist beispielsweise fraglich, ob für alle sprachlichen Ausdrücke im Sinne des monosemic approach eine minimale Grundbedeutung gefunden werden kann, von der sich

\footnotetext{
„vage, insoweit es in der Schwebe bleibt, wie weit ein Ding auf dem Spektrum nach unten - in Richtung Gelb - und wie weit es nach oben - in Richtung Blau - liegen und immer noch als grün gelten kann“(Quine 1960: 223).

Vor dem Hintergrund, dass mit dem Terminus Vagheit auf sehr unterschiedliche Phänomene referiert wird, wird nachfolgend auf diesen Begriff verzichtet und stattdessen einzig auf den Begriff der Monosemie zurückgegriffen.

78 Zunehmend werden in die Diskussion, ob sich die verschiedenen Verwendungsmöglichkeiten eines Ausdrucks auf eine (abstrakte) Grundbedeutung zurückführen lassen oder ob man besser mehrere eigenständige ggf. miteinander verknüpfte Verwendungsweisen annehmen sollte, auch psycholinguistische und psychologische Studien einbezogen. Der interessierte Leser sei hier u. a. auf die Untersuchungen von Sandra/Rice (1995) und Rice (1999) verwiesen. Die in Bons (2009) dargestellten, z.T. gegensätzlichen Ergebnisse einiger Studien zeigen jedoch, dass der Versuch, auf der Basis psycholinguistischer Experimente Aussagen zu möglichen mentalen Konzepten o. Ä. machen zu wollen, mit Vorsicht zu genießen ist, „was sowohl dagegen spricht, Zusammenhänge zwischen kognitiven Strukturen und semantischen Strukturen uneingeschränkt zu postulieren, als auch dagegen, sie voreilig zu bestreiten“ (ebd. 22).
} 
alle weiteren Verwendungsweisen des Lexems ableiten lassen. Ein Beispiel hierfür liefert Taylor (1995b: 14f.); anhand des Verbs to leave zeigt er, dass die Bedeutungen sprachlicher Ausdrücke häufig so verschieden sind, dass die Bestimmung einer zugrundeliegenden abstrakten Grundbedeutung, für die über den (sprachlichen) Kontext alle notwendigen semantischen Spezifikationen gegeben werden können, schwierig ist (vgl. Evans 2004: 89). Darüber hinaus müsste, um alle möglichen Verwendungsweisen eines Ausdrucks erfassen zu können, die anzunehmende Minimalbedeutung der meisten sprachlichen Ausdrücke so abstrakt sein, dass der Unterschied zu anderen Ausdrücken mit ähnlichen Verwendungsweisen z. T. verdeckt würde. Egg (1994) verweist in diesem Zusammenhang auf das Verb to consider, dessen Grundbedeutung kognitive Aktivität eines Menschen, deren Objekt eine Information ist auch die Grundbedeutung der Verben to think oder to reflect sein könnte (vgl. ebd. 10). Ebenfalls problematisch ist die Annahme, dass sich die unterschiedlichen Lesarten eines sprachlichen Ausdrucks - basierend auf einer einheitlichen, abstrakten Basisbedeutung - ausschließlich als Resultat seiner konzeptuellen Differenzierung im Kontext ergeben (vgl. Dölling 2005). Das Beispiel in (76) zeigt, dass die Bedeutung des Satzes - bei gleichbleibendem (sprachlichem) Kontext - ausschließlich durch den Austausch der Präposition verändert wird:

(76) a. John ran up the stairs. (nach Evans 2004: 89)

b. John ran down the stairs. (ebd.)

Beispiele wie diese zeigen, dass

while context is clearly important in meaning-construction and interpretation, at least some of the meanings associated with words must derive from particular lexical concepts being paired in semantic memory with particular lexical forms. Put another way, some of the meaning derived from a particular utterance must be associated with words, as must be the case in examples such as $((76), \mathrm{TvdB})[. .$.$] , in which a different meaning results when a$ particular lexical item is swiched, in this case up for down.

(Evans 2004: 89)

Eine weitere Schwierigkeit der Monosemie-Hypothese besteht darin, dass die Kenntnis einer abstrakten Minimalbedeutung im Sinne des monosemic approach häufig nicht ausreicht, um einen sprachlichen Ausdruck in konkreten Kommunikationssituationen richtig zu verwenden bzw. $\mathrm{zu}$ verstehen. Es erscheint unwahrscheinlich, dass die - im Sinne des monosemic approach nicht im mentalen Lexikon gespeicherten (vgl. Taylor 1995b: 13) - Bedeutungsvarianten eines Lexems in der jeweiligen Kommunikationssituation ad hoc und immer wieder neu aus einer abstrakten Grundbedeutung abgeleitet werden müssen. Vielmehr ist es wahrscheinlicher, dass man die Bedeutungen eines sprachlichen Ausdrucks nach 
und nach lernt, indem man seine Gebrauchsweisen verinnerlicht ${ }^{79}$ (vgl. u. a. Fritz 1995: 95; Gloning 1996: 208f.). Des Weiteren stellen sich die Fragen, wie bei der Annahme einer abstrakten Basisbedeutung die historisch unterschiedlichen Verwendungsspektren eines Ausdrucks zu erfassen sind (vgl. Taylor 1995b: 21f.) und warum bei Übernahme eines fremdsprachlichen Ausdrucks häufig nur einzelne Verwendungsweisen aufgenommen werden (vgl. ebd. 22).

Doch nicht nur die Monosemie-, sondern auch die Homonymie-Hypothese ist mit zahlreichen Problemen verbunden. So nimmt der homonymic approach für jede Verwendungsweise eines sprachlichen Ausdrucks einen eigenen Eintrag im mentalen Lexikon an, wobei keinerlei Verbindungen zwischen den unterschiedlichen Verwendungsweisen bestehen. Diese Annahme wird jedoch durch die zahlreichen Untersuchungen, die eine hochstrukturierte Organisation des mentalen Lexikons nachweisen (vgl. u. a. Quillian 1968; Baayen 2007; Aitchinson $2012^{4}$ ), widerlegt. Eine strikte Trennung der Verwendungsweisen spricht darüber hinaus auch gegen die zahlreichen Befunde der diachronischen und synchronischen Sprachforschung, wonach der homonymic approach keine Rückschlüsse darauf zulässt, warum sprachliche Ausdrücke eher mit neuen, unterschiedlichen Bedeutungen verknüpft werden, als dass sich eine neue phonologische Form ausbildet (vgl. Evans 2004: 88).

Da beide Extrempositionen mit Schwierigkeiten verbunden sind, werden in dieser Arbeit die verschiedenen Verwendungsmöglichkeiten eines sprachlichen Ausdrucks weder auf eine Ursprungsbedeutung bzw. eine relativ abstrakte Bedeutungsrepräsentation zurückgeführt, von der Varianten konversationell abgeleitet werden müssen (monosemic approach), noch wird davon ausgegangen, dass die einzelnen Verwendungsweisen völlig unabhängig voneinander repräsentiert werden (homonymic approach). Stattdessen wird hier im Sinne des polysemic approach (vgl. u.a. Cuyckens/Zawada 2001; Evans 2004; Evans 2005) die Auffassung vertreten, dass eine linguistische Form mit einer Vielzahl unterschiedlicher, aber miteinander verwandter Bedeutungen verbunden ist (vgl. Evans 2005: 33). Zwar fühle ich mich keinem der in diesem Zusammenhang diskutierten Ansätze im Detail verpflichtet, teile jedoch die Meinung, dass die einzelnen Verwendungsmöglichkeiten damit nicht isoliert nebeneinander stehen, sondern zumindest einige von ihnen miteinander verknüpft sind bzw. gewisse Verwandtschaften zwischen einander ausweisen (vgl. Tyler/Evans 2003: 7). So ist davon

79 Das bedeutet natürlich nicht, dass alle möglichen Bedeutungen eines Ausdrucks auch tatsächlich im mentalen Lexikon gespeichert sind. Vielmehr gilt, dass ,at least some of the senses could be generated on-line, in accordance with general principles of meaning extension“ (Taylor 2003a: 642). 
auszugehen, dass das Lexikon keine unsystematische Ansammlung unverbundener Lexeme ist; vielmehr zeichnet es sich durch eine hohe Systematik und Produktivität aus (vgl. Evans 2005: 34). Mit dieser Annahme ist allerdings nicht die Bedingung verbunden, dass

grundsätzlich alle Verwendungsweisen miteinander verknüpft sind oder dass es eine „Kern“-Verwendungsweise bzw. eine übergeordnete allgemeine Verwendungsweise geben muss, von der alle Verwendungsweisen abgeleitet werden können.

(Bons 2009: 9)

Vielmehr können die Verwendungsspektren polysemer Ausdrücke auch familienähnliche Strukturen im Sinne Wittgensteins (2001) aufweisen. Das Konzept der Familienähnlichkeit ${ }^{80}$ geht von der Annahme aus,

dass eine Familie aus mehreren Mitgliedern besteht, von denen jedes Mitglied Ähnlichkeitsbeziehungen zu mehreren anderen Mitgliedern derselben Familie unterhält, aber nicht notwendigerweise zu allen anderen Mitgliedern der Familie. Außerdem existiert kein einzelnes Merkmal, das allen Mitgliedern einer Familie gemeinsam sein muss.

(Proost/Winkler 2015: 8f.)

Die Idee, dass sprachliche Ausdrücke - hier Verben - verschiedene Verwendungsarten aufweisen, die durch Familienähnlichkeiten miteinander verbunden und möglicherweise um einen Prototypen gruppiert sind (vgl. Cuyckens/Zawada 2001: xiii), ist für die nachfolgenden Überlegungen insofern von Bedeutung, als meiner Auffassung nach der „Valenzgrundsatz, dass das Verb Zahl und Art der Ergänzungen bestimmt, [...] nur aufrecht zu erhalten [ist, TvdB], wenn von Verblesarten ausgegangen wird“ (Ágel/Fischer2010: 266). Will man also Aussagen zu den in F1 und F2 formulierten Forschungsfragen treffen, müssen zunächst die unterschiedlichen Verwendungsweisen der Verben ermittelt werden ${ }^{81}$. Detaillierte Überlegungen dazu, wie sich die einzelnen Verwendungsweisen der hier

80 Das Konzept der Familienähnlichkeit lässt sich auf unterschiedliche Bereiche der semantischen Untersuchung anwenden, „u. a. auf die Verwendungen eines Ausdrucks, die einander ggf. familienähnlich sind und die man bei einer größeren Ähnlichkeit vermutlich eher zu einer Gruppe zusammenfassen wird, und auf die Verwendungsweisen eines Ausdrucks, deren Familienähnlichkeiten die semantische Struktur des Verwendungsspektrums konstituieren können“ (Bons 2009: 138).

81 Wenn auch im Folgenden im Sinne der Polysemie-Hypothese davon ausgegangen wird, dass es sich bei den verschiedenen Verwendungsmöglichkeiten eines sprachlichen Ausdrucks um mehrere eigenständige, miteinander verwandte Verwendungsweisen handelt, bedeutet das nicht, dass grundsätzlich „keine Unterscheidung von konversationellen Verwendungen und konventionellen Verwendungsweisen gemacht würde“ (Bons 2009: 23). So werden etwa rein okkasionelle Verwendungen keineswegs als eigenständige Verwendungsweisen, die im Lexikon zu erfassen sind, betrachtet. 
untersuchten Verben nach möglichst objektivierbaren Kriterien ermitteln lassen, finden sich in Kapitel 4; konkrete einzelverbbezogene Umsetzungen dieser Methoden folgen in Kapitel 5.

\subsection{Bedeutungsvarianten und Valenz}

Bereits in frühen Arbeiten zur Valenz im Allgemeinen sowie zur Argumentweglassung im Speziellen finden sich wiederholt Aussagen, nach denen es sinnvoll sei, z. B. im Zusammenhang mit der hier zu untersuchenden Komplementweglassung „lieber von polysemen Verben oder Bedeutungsvarianten eines Verbs mit unterschiedlichen Valenzstrukturen“ zu sprechen (Breindl 1989: 18) - insbesondere dann, wenn wie in den nachfolgenden Beispielen „fehlende Ergänzbarkeit zur „vollen“ Valenz der a-Strukturen in den b-, c- und d-Fällen“ (ebd.) vorliegt:

(77) a. Ein Hund ist in die Schlucht gefallen! (ebd.; Hervorhebung getilgt, TvdB)

b. Hongkongbank-Aktien weiter gefallen! (ebd.)

c. Unser Vater ist bei El-Alamein gefallen! (ebd.)

d. Die Entscheidung ist gefallen! (ebd.)

(78) a. Hänschen geht in die Schwimmschule. (ebd.; Hervorhebung id.)

b. Geht die Uhr noch? (ebd.)

c. Wie geht's? - Danke, es geht. (ebd.)

Eine konsequente Umsetzung der geforderten Lesartendifferenzierung fand jedoch in der Literatur häufig nicht oder nur unzureichend statt. Stattdessen liegen bis heute Darstellungen, in denen von einer einfachen Interpretation im Sinne von ,1 Verb = 1 Valenzträger' ausgegangen wird, vor ${ }^{82}$.

Ein solches Vorgehen führt m. E. regelmäßig $\mathrm{zu}$ verzerrten Valenzangaben. Darüber hinaus kommt es dadurch besonders vor dem Hintergrund der im Rahmen dieser Arbeit zu untersuchenden Forschungsfragen immer wieder zu unscharfen und nicht überzeugenden Aussagen zur Argumentweglassung im Allgemeinen sowie möglichen semantischen Konsequenzen im Speziellen. In der Forschungsliteratur wird etwa das Verb akzeptieren als Beispiel einer

82 Erkennbar wird dies m. E. daran, dass die Autoren eine explizite Lesartendifferenzierung nicht vornehmen. Stattdessen verwenden sie Formulierungen, die eine Gleichsetzung von ,1 Verb =1 Valenzträger ' suggerieren. Aussagen wie die folgenden belegen dies: „die semantische Valenz von einwilligen“ (Jacobs 1994b: 300) oder „Sie setzt aber voraus, dass das Lexikon dem Verb unterschreibt neben [...]“ (Jacobs 2003: 393). 
sortal spezifischen Interpretation impliziter Argumente angeführt. Nach Jacobs (1994b: 302) erweist sich das implizite direkte Objekt der valenzreduzierten Variante von akzeptieren dabei insofern als spezialisiert, als es ,auf eine enger begrenzte Sorte von Referenten festgelegt [...] [ist, TvdB] als entsprechende Ergänzungen der Normalvariante des [...] Valenzträgers“. In diesem Sinne kann nach Aussage des Autors ein valenzreduzierter Satz wie Er hat akzeptiert nicht als Er hat seine Mitmenschen/seine Behinderung akzeptiert verstanden werden. Auch Engelberg weist auf diese Beobachtung hin, indem er schreibt, dass für das Verb akzeptieren „die Interpretationsbeschränkungen für implizite Argumente strenger sind als die entsprechenden Selektionsrestriktionen für die expliziten Argumente der transitiven Verbvariante“ (Engelberg 2003: 64). Transitives akzeptieren referiere auf Vorschläge, Pläne, Angebote, Situationen, Schicksale und Krankheiten, wohingegen in der intransitiven Variante des Verbs nur noch Vorschläge, Pläne und Angebote, nicht aber Situationen, Schicksale und Krankheiten interpretativ verfügbar seien (vgl. ebd.). Entsprechende Beispiele scheinen die Annahmen von Jacobs und Engelberg zu bestätigen; so ist Argumentweglassung in (79a) - mit Bezug auf einen konkreten Vorschlag problemlos möglich, wohingegen sie in (79b) - mit Bezug auf ein persönliches Schicksal - ausgeschlossen ist:

(79) a. Von dem Vorschlag, dem ganzen Haufen erschöpfter Lexikographen doch mal einen Forschungsaufenthalt auf Kreta zu finanzieren, war Wolf begeistert, und er akzeptierte sofort/und er akzeptierte den Vorschlag sofort. (nach Engelberg 2003: 64)

b. Nun war es also Wolfs Schicksal, sein halbes Leben mit einem Haufen manischer lexikographischer Zettelschreiber $\mathrm{zu}$ verbringen, *aber er akzeptierte sofort/aber er akzeptierte dieses Schicksal sofort. (ebd.)

Vor dem Hintergund, dass sich die Objekt-NP von akzeptieren in der valenzreduzierten intransitive Verwendung im Unterschied zur transitiven Verwendung nur auf Vorschläge, Pläne oder ähnliche Referente beziehen kann (vgl. Engelberg 2002: 373f.), gehen Jacobs und Engelberg davon aus, dass das Verb zwei mit spezifischen Restriktionen für ihre jeweiligen - expliziten bzw. impliziten - Komplemente verbundene Varianten aufweist (vgl. ebd. 374). Die enge semantische Verbindung der beiden Valenzvarianten kann ihrer Auffassung nach durch ein Bedeutungspostulat (80c) erfasst werden (vgl. ebd. 373):

(80) a. akzeptieren

SynVal: $\quad /$ nom $^{\mathrm{y}} / \mathrm{akk}^{\mathrm{x}}$

SemVal: $\quad \lambda x \lambda y[\operatorname{AKZEPTIER~}(\mathrm{x})(\mathrm{y})]$ 
b. akzeptieren a

SynVal: $\quad /$ nom $^{\mathrm{y}}$

SemVal: $\quad \lambda y\left[\operatorname{AKZEPTIER}_{\mathrm{R}}(\mathrm{x})(\mathrm{y})\right]$

c. BR: $\quad \forall x \forall y\left[\operatorname{AKZEPTIER}_{\mathrm{R}}(\mathrm{x})(\mathrm{y}) \rightarrow \operatorname{AKZEPTIER}_{(\mathrm{x})}(\mathrm{y})\right]$

Inwieweit es sich bei den hier beschriebenen Interpretationsbesonderheiten der impliziten Argumente von akzeptieren jedoch tatsächlich um aus Argumentweglassung resultierende semantische Spezifizierungen handelt, ist m. E. fraglich. Winkler (2009: 4) weist darauf hin, dass das Verb zwei unterschiedliche Lesar-

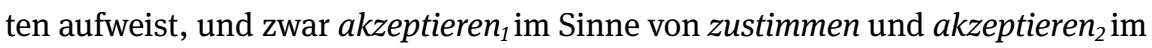
Sinne von hinnehmen. Die in Kapitel 4 dargestellten Methoden zur Lesartendifferenzierung sowie die in Kapitel 5.1.2.1 durchgeführten Einzelverbuntersuchungen zu akzeptieren werden zeigen, dass tatsächlich beide akzeptieren-Lesarten - neben weiteren, von Winkler nicht angegebenen Verwendungsweisen - anzunehmen sind. Die Unterschiede der einzelnen Verwendungsweisen manifestieren sich dabei in den je spezifischen semantischen und syntaktischen Valenzrahmen.

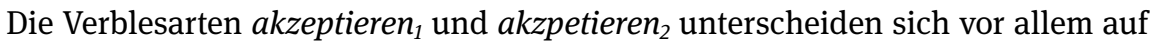
der semantischen Ebene ${ }^{83}$ : Während akzeptieren ${ }_{1}$ aufgrund der darin enthaltenen Implikation einer bewussten Einflussnahme, Entscheidung oder Wahlmöglichkeit dessen, womit man sich einverstanden erklärt, als Objekte nur solche zulässt, die bewusst angenommen oder abgelehnt werden können - etwa Vorschläge, (Gesetzes-)Entwürfe, Angebote usw. - können bei akzeptieren ${ }_{2}$ aufgrund der fehlenden Möglichkeit einer bewussten Einflussnahme als potenzielle Argumente nur Objekte verwendet werden, die gerade nicht beeinflussbar sind - z. B. eine Behinderung, eine Krankheit, eine Entscheidung oder ein Urteil.

Zudem zeichnen sich beide akzeptieren-Lesarten durch unterschiedliche

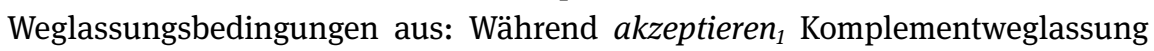
problemlos erlaubt und damit die in (81b) dargestellte R-Valenz anzunehmen ist, ist sie bei akzeptieren ${ }_{2}(82)$ ausgeschlossen:

a. akzeptieren $_{1}$

\begin{tabular}{|c|c|}
\hline i.S.v. & zustimmen \\
\hline SynVal: & $/ \operatorname{nom}^{\mathrm{y}} / \mathrm{akk}^{\mathrm{x}}$ \\
\hline SemVal: & $\lambda x \lambda y\left[\operatorname{AKZEPTIER}_{1}(\mathrm{x})(\mathrm{y})\right]$ \\
\hline BR1: & $\forall \mathrm{x} \forall \mathrm{y}\left[\operatorname{AKZEPTIER}_{1}(\mathrm{x})(\mathrm{y}) \rightarrow\right.$ EINVERSTANDEN (x) (y)] \\
\hline BR2: & 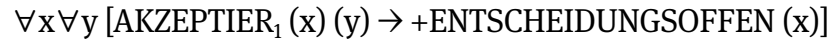 \\
\hline
\end{tabular}

83 Auf der syntaktischen Ebene verhaltenen sich beide Verbverwendungen zunächst gleich; beide weisen die syntaktische Valenz /nom/akk auf. 
b. akzeptieren ${ }_{1 \_R}$

SynVal: $\quad /$ nom $^{\mathrm{y}}$

SemVal: $\quad \lambda y\left[\operatorname{AKZEPTIER}_{1 \_R}(\mathrm{x})(\mathrm{y})\right]$

BR1: $\quad \forall \mathrm{x} \forall \mathrm{y}\left[\right.$ AKZEPTIER $_{1 \_\mathrm{R}}(\mathrm{x})(\mathrm{y}) \rightarrow$ IN S BEKANNT(x)]

BR2: $\quad \forall x \forall y\left[\operatorname{AKZEPTIER}_{1 \_\mathrm{R}}(\mathrm{x})(\mathrm{y}) \rightarrow \operatorname{AKZEPTIER}_{1}(\mathrm{x})(\mathrm{y})\right]$

(82) akzeptieren 2

i.S.v. hinnehmen

SynVal: $\quad /$ nom $^{\mathrm{y}} / \mathrm{akk}^{\mathrm{x}}$

SemVal: $\quad \lambda \mathrm{x} \lambda \mathrm{y}\left[\operatorname{AKZEPTIER}_{2}(\mathrm{x})(\mathrm{y})\right]$

BR1: $\quad \forall x \forall y$ [AKZEPTIER 2 ( $\quad$ (y) $\rightarrow \operatorname{HINNEHMEN~(x)~(y)]~}$

BR2: $\quad \forall \mathrm{x} \forall \mathrm{y}\left[\operatorname{AKZEPTIER}_{2}(\mathrm{x})(\mathrm{y}) \rightarrow\right.$-ENTSCHEIDUNGSOFFEN(x)]

Diesen Annahmen folgend, lassen sich die in (79) beobachteten Weglassungsunterschiede ohne Schwierigkeiten den beiden hier dargestellten akzeptieren-Lesarten zuweisen: In (79a) bezieht sich akzeptieren auf einen entscheidungsoffenen

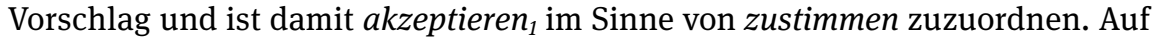
diese Weise interpretiert lässt akzeptieren Argumentweglassung problemlos zu. In (79b) rekurriert akzeptieren hingegen auf ein nicht beeinflussbares Schicksal.

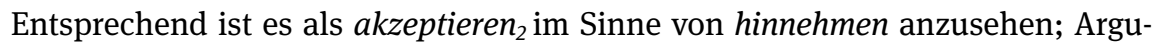
mentweglassung ist entsprechend ausgeschlossen.

Bei den beobachtbaren und in der Literatur beschriebenen semantischen Effekten impliziter Argumente handelt es sich demnach nicht um Interpretationsbesonderheiten stellenreduzierter Alternativvalenzen im Vergleich zu den nicht-stellenreduzierten Valenzen derselben Lesarten. Vielmehr sind die stellenreduzierten Alternativvalenzen einer von mehreren Lesarten (hier akzeptieren $_{1}$ ) zuzuordnen, von denen sie die sortalen Besonderheiten übernehmen.

Überlegungen wie diese machen $\mathrm{m}$. E. deutlich, dass die bislang angenommene und immer wieder praktizierte einfache Gleichsetzung ,1 Valenzträger $=1$ Verb' nicht aufgeht. Mit Blick auf die in F2 formulierte Frage wird im weiteren Verlauf dieser Arbeit davon ausgegangen, dass die meisten Verben eine Vielzahl unterschiedlicher Lesarten aufweisen. Es zeigt sich, dass „in der Regel jede Lesart eines Verbs in einem eigenen Spektrum von Argumentstrukturmustern auftritt, und nicht jedes Argumentstrukturmuster auch mit jeder Lesart eines Verbs möglich ist“ (Winkler 2009: 4).

Zur Darstellung eines polysemen Ausdrucks wird im Folgenden auf die Strategie der „lexikalischen Spezifikation“ (vgl. Dölling 2005: 174) zurückgegriffen. Unter lexikalischer Spezifikation versteht man „die Menge der linguistischen Informationen, die mit einem Lexikoneintrag gekoppelt sind“(Bahns 1997: 169). Entsprechend definiert die „mit einem lexikalischen Zugriffselement verbundene Spezifikation 
[...] die linguistischen Aspekte eines Lexikoneintrags“ (Handtke 1997²: 92). Hierzu gehören neben phonologischen Merkmalen (Phonemstruktur, Silben- und Akzentstruktur, Intonationsmuster) und morphologischen Eigenschaften (Flexionsmuster, Derivations- und Kompositionsschemata) vor allem auch syntaktische Informationen (Argumentstruktur, syntaktische Idiosynkrasien) und semantisch/ konzeptionelle Aspekte (Bedeutung, semantische Relationen) (vgl. ebd. 93). Für die nachfolgenden Überlegungen spielen vor allem die syntaktischen und konzeptionellen Spezifikationen eines Lexikoneintrags eine herausragende Rolle. So unterscheiden sich die verschiedenen Lesarten eines Verbs, ,zum einen durch die Anzahl der alternativen syntaktischen Subkategorisierungen des Verbs und zum anderen durch die der alternativen sortalen Restriktionen für seine Argumente“ (Dölling 2005: 174). Die spezifischen syntaktischen und semantischen Forderungen und Restriktionen der einzelnen Verbverwendungen werden im Sinne des hier vertretenen multidimensionalen Valenzkonzepts mit Hilfe der syntaktischen und semantischen Valenz angegeben. Der Lexikoneintrag einer jeden Verblesart besteht aus mindestens zwei Angaben, wobei SynVal die syntaktische Subkategorisierung spezifiziert und damit die Komplementstruktur der Verblesart festlegt, während SemVal die Argumentstruktur der Verblesart angibt und über eine Menge von Bedeutungspostulaten die Vorkommensbeschränkungen der jeweiligen Argumente (Selektionsrestriktionen) spezifiziert. Ausführliche Überlegungen hierzu finden sich in Kapitel 2.

Zusätzlich wird die Bedeutung der angenommenen Verblesarten mit Hilfe von Bedeutungsparaphrasen ${ }^{84}$ (im Folgenden auch BP) angegeben; ,by means of another word, or group of words, whose meaning is supposedly synonymous with the meaning being defined, or in the form of a description of the kind of things the word can be used to refer to" (Taylor 2003a: 641) wird versucht, die Bedeutung der Lesart zu umschreiben. So können die vom Verb eröffneten Stellen, die bedeutungskonstitutiv für die betreffende Verblesart sind, sichtbar gemacht werden (vgl. Primus 2015: 79). Es wird in Kauf genommen, dass die Paraphrasemethode mit zahlreichen Schwierigkeiten ${ }^{85}$ verbunden ist - vgl. etwa das Problem, dass Bedeutungsparaphrasen häufig nur dann verstanden werden können, wenn bereits auf Weltwissen basierende Vorstellungen zu der beschriebenen Lesart und ihren Verwendungsmöglichkeiten existieren und die zur Paraphrase herangezogenen Ausdrücke i.d.R. selbst wieder polysem sind.

84 Andere, hier nicht weiter berücksichtigte Möglichkeiten zur Bedeutungsfestlegung sind framebasierte Ansätze (vgl. z. B. Martin (2001)) sowie piktographische Repräsentationen (vgl. u. a. Huumo (2001)).

85 Diese Schwierigkeiten werden in Miller/Leacock (2001) ausführlich dargestellt. 
Exemplarisch sei dies hier an drei der in (75) dargestellten stellen-Lesarten im Einzelnen stellen ${ }_{2}$ stellen $_{5}$ und stellen $_{8}-$ verdeutlicht $^{86}$ :

(83) stellen $_{2}$

$\mathrm{BP}$ :

jemand/etwas stellt etwas an einen bestimmten Platz, bringt es an eine bestimmte Stelle (vgl. DUDEN 2012 __stellen)

Bsp.: $\quad$ Rudolf hat ein Glas auf den Tisch gestellt. (TvdB)

SynVal: $\quad /$ nom $^{\mathrm{z}} / \mathrm{akk}^{\mathrm{y}} / \emptyset^{\mathrm{x}}$

SemVal: $\quad \lambda x \lambda y \lambda z\left[\operatorname{STELL}_{2}(\mathrm{x})(\mathrm{y})(\mathrm{z})\right]$

BR: $\quad \forall \mathrm{x} \forall \mathrm{y} \forall \mathrm{z}\left[\mathrm{STELL}_{2}\right.$ (x) (y) (z) $\rightarrow{ }_{\text {dir }} \mathrm{ORT}(\mathrm{x})$ \& KONKRET(y) \& $\operatorname{BELEBT}(\mathrm{z})]$

(84) stellen $_{5}$

BP:

jemand/etwas sorgt dafür, dass jemand/etwas zur Stelle ist; bereitstellen (vgl. DUDEN $2012^{4}$ _stellen)

Bsp.: Die Universität hat die Räume gestellt. (TvdB)

SynVal: $\quad /$ nom $^{\mathrm{y}} / \mathrm{akk}^{\mathrm{x}}$

SemVal: $\quad \lambda x \lambda y\left[\operatorname{STELL}_{5}(\mathrm{x})(\mathrm{y})\right]$

BR: $\forall \mathrm{x} \forall \mathrm{y}\left[\operatorname{STELL}_{5}(\mathrm{x})(\mathrm{y}) \rightarrow \operatorname{KONKRET}(\mathrm{x}) \& \operatorname{PERSON}(\mathrm{y}) \mathrm{V}\right.$ INSTITUTION(y)]

(85) stellen $_{8}$

BP:

jemand/etwas zwingt jemanden/etwas zum Stehenbleiben und bekommt ihn/es dadurch in seine Gewalt (vgl. DUDEN 2012 ${ }^{4}$ stellen)

Bsp.: $\quad$ Die Polizei hat den Dieb gestellt. (TvdB)

SynVal: $\quad /$ nom $^{\mathrm{y}} / \mathrm{akk}^{\mathrm{x}}$

SemVal: $\quad \lambda y \lambda x\left[\operatorname{STELL}_{8}(\mathrm{x})(\mathrm{y})\right]$

BR: $\quad \forall x \forall y$ [STELL $_{8}$ (x) (y) $\rightarrow$ PERSON(x) V TIER(x) V

FAHRZEUG(x) \& PERSON(y) V

INSTITUTION(y) V TIER(y)]

Die Darstellungen in (83) bis (85) veranschaulichen, dass die einzelnen Bedeutungsvarianten der Verben mit je spezifischen Valenzrahmen verbunden sind. Die Unterschiede manifestieren sich dabei sowohl auf der syntaktischen - etwa

86 Eine exakte Beschreibung der Semantik der Aktanten sowie exakte Semanalysen der Valenzträger sind in der vorliegenden Arbeit nicht zu leisten. Entsprechend stellen die hier und nachfolgend dargestellten Strukturen nur mögliche Auszüge aus den semantischen Valenzen der Verblesarten dar. 
durch abweichende Realisierungs- und Merkmalsforderungen - als auch auf der semantischen Ebene - z. B. durch divergierende Selektionsrestriktionen.

Der Bedeutungsparaphrase ${ }^{87}$ in (83) ist zu entnehmen, dass das Verb stellen 2 die Realisierung von drei Verbbegleitern fordert. Dieser Anspruch geht einher mit spezifischen Merkmalsforderungen für die einzelnen Argumente; so muss das erste Dependens im Nominativ und das zweite im Akkusativ realisiert werden. Der dritten Ergänzung werden dagegen keine Forderungen bezüglich ihrer Form auferlegt (angezeigt durch das KR-Valenzmerkmal /Ø), vgl. Rudolf hat ein Glas auf den Tisch/neben das Sofa/unter den Schrank gestellt. Dass dennoch nicht beliebige Ausdrücke die entsprechende Valenzstelle füllen können, folgt aus dem in der semantischen Valenz enthaltenen Postulat, wonach es sich bei einer möglichen Ergänzung um ein Prädikat handeln muss, das den Zielort des stellenVorgangs näher beschreibt. Ebenfalls aus der semantischen Valenz des verbalen Valenzträgers ergeben sich die Forderungen nach Belebtheit der die Handlung des Stellens ausführenden Person sowie nach Konkretheit des der Handlung des Stellens unterworfenen Relats.

Anders verhält es sich dagegen bei stellen ${ }_{5}$ - dargestellt in (84). Der Bedeutungsparaphrase entsprechend beansprucht stellen $_{5}$ die Realisierung von zwei Verbbegleitern, davon einen im Nominativ und einen im Akkusativ. Mit diesen kategorialen Valenzforderungen gehen ebenfalls spezifische, in der semantischen Valenz des Verbs enthaltene Forderungen einher; das Verb stellen ${ }_{5}$ beschreibt etwa eine Situation, in der eine Person bzw. eine Institution jemandem etwas Konkretes zur Verfügung stellt.

Auch das in (85) wiedergegebene stellen $_{8}$ fordert die Realisierung eines Nominativ- und eines Akkusativdependens. Die mit der semantischen Valenz des Valenzträgers verbundenen Forderungen machen jedoch den Unterschied zwischen stellen ${ }_{5}$ und stellen ${ }_{8}$ deutlich; so postuliert stellen, ${ }_{8}$ dass es sich bei dem die Handlung ausführenden Relat um eine Person bzw. Institution (z. B. Polizei) handelt, während es sich bei dem der Handlung unterworfenen Relat um eine Person, ein Tier oder ein Fahrzeug handeln muss.

Zusammenfassend bleibt festzuhalten, dass Valenzinformationen - und damit auch Angaben zur Argumentweglassung sowie möglichen hieraus resultierenden

87 Die in lexikalischen Repräsentationen verwendeten Paraphrasen sind nur dann weitgehend synonym zu den zu paraphrasierenden Ausdrücken, wenn sie ihnen auch kategorial entsprechen. Dass das in den Beispielen in (83) bis (85) nicht der Fall ist - die Paraphrasen entsprechen Sätzen und keinen Verben oder Verbalphrasen - wird hier und im Folgenden zugunsten einer einheitlicheren Darstellungsweise in Kauf genommen und stattdessen auf die in Wörterbüchern gängige Praxis der Bedeutungsumschreibung zurückgegriffen (vgl. u. a. Duden, E-VALBU). 
semantischen Konsequenzen - immer lesartenbezogen anzugeben bzw. zu interpretieren sind.

Obwohl auf die Notwendigkeit einer Lesartendisambiguierung in der Literatur immer wieder hingewiesen wurde (vgl. z. B. Fillmore 1986: 99; Jacobs 1994a: 14; Ágel 2000: 115ff.; Primus 2015: 88 - FN6), wurde sie in der Praxis häufig nicht oder nur unzureichend umgesetzt. Fehlende bzw. unspezifische Lesartenangaben haben dabei immer wieder dazu geführt, dass es zu ungenauen Valenzangaben sowie unscharfen oder nicht überzeugenden Aussagen zur Argumentweglassung und ihren möglichen semantischen Konsequenzen kam (s. o.).

Als besonders problematisch erweisen sich fehlende Lesartendifferenzierungen vor dem Hintergrund der hier angestrebten Untersuchungen, da in den meisten bisherigen Arbeiten zur Komplementweglassung sowie daraus resultierenden semantischen Effekten i.d.R. nicht ersichtlich wird, welche Verblesart die Autoren ihren Analysen zugrunde gelegt haben. Zwar machen die meisten Autoren, die semantische Spezifizierungen impliziter Argumente untersuchen, Angaben zur jeweiligen Spezifizierung der impliziten Ergänzungen; Informationen dazu, welcher Art die explizit realisierten Objekt-Argumente vor Argumentweglassung sein müssen, werden jedoch nur selten gegeben. Auch hierdurch bleibt häufig unklar, welche Verblesart die Autoren ihrer Untersuchung zugrunde legen und ob die Lesart des untersuchten Verbs vor und nach Argumentweglassung stabil bleibt.

Im folgenden Kapitel 4 wird der Frage nachgegangen, wie die unterschiedlichen Lesarten der hier zu untersuchenden Verben ermittelt werden können. 